\title{
DISCUSSION
}

\section{Tensiometer saturation and the reliable measurement of soil suction}

\author{
W. A. TAKE and M. D. BOLTON (2003). Géotechnique 53, No. 2, 159-172
}

\section{A. Tarantino, Università degli Studi di Trento, Italy}

The authors address two important issues relating to tensiometer measurements: the initial saturation of the high airentry ceramic and the unreliable response of the tensiometer due to the inadequate saturation.

Ridley \& Burland (1999) suggested a procedure for initial saturation where the first stage consisted of removal of air from the ceramic. This was achieved by applying a vacuum in a chamber partly filled with de-aired water. The authors assume that the ceramic may adsorb water molecules during porous ceramic installation and evacuation, and this is likely to reduce the wettability of the ceramic during the subsequent inundation. To cope with this problem, the authors propose a new initial saturation process where air removal is completed in two steps: oven-drying and evacuation in the absence of water.

A very similar procedure has already been suggested by Tarantino \& Mongiovì (2002) using the vacuum chamber shown in Fig. 15. The only difference lies in the drying of the ceramic, as the tensiometer was placed in a desiccator containing silica gel and not in an oven. The use of this double-compartment vacuum chamber was to overcome another supposed limitation of the procedure proposed by Ridley \& Burland (1999). As free water is present in the vacuum chamber, water vapour continuously develops during evacuation. This prevents the attainment of very low absolute pressures, and hence reduces the effectiveness of air removal from the ceramic.

The authors have shown that the initial saturation was sufficient to saturate the tensiometer fitted with the $100 \mathrm{kPa}$ air-entry porous ceramic. However, the initial saturation alone was not sufficient to saturate the ceramic with a higher air-entry value $(300 \mathrm{kPa})$, and a pressurisation was required to measure water tension. Similarly, the discusser found that initial saturation alone was not sufficient to saturate the Trento tensiometers, which use a ceramic with a very high air-entry value $(1500 \mathrm{kPa})$. After saturation using the saturation chamber shown in Fig. 15 and a vacuum pump capable

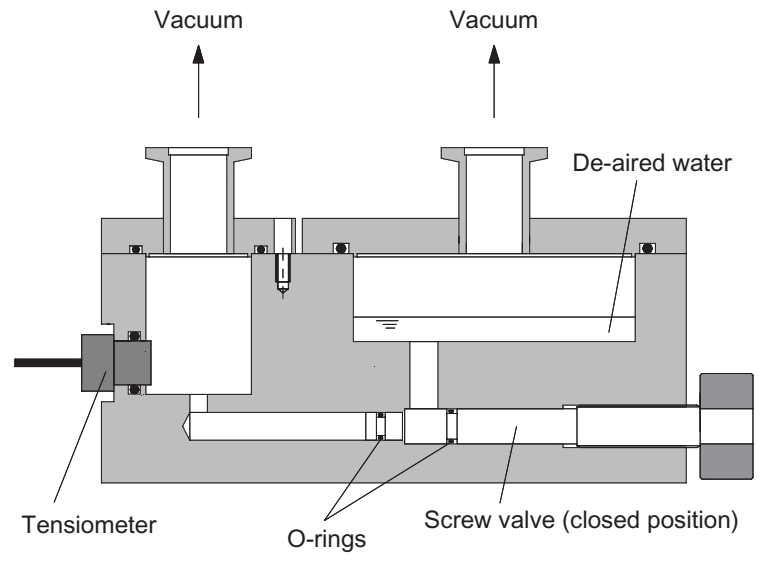

Fig. 15. Schematic layout of the Trento double-compartment vacuum chamber of a measured minimum absolute pressure of $0.03 \mathrm{kPa}$, the tensiometers could withstand a minimum gauge pressure of about $-300 \mathrm{kPa}$ for only 1 or $2 \mathrm{~s}$.

It is then possible that the initial saturation adopted by the authors and the discusser does not significantly enhance the procedure proposed by Ridley \& Burland (1999). The discusser has saturated two Imperial College tensiometers following the procedure suggested by Ridley \& Burland (1999), and using a large triaxial cell as a saturation chamber and a vacuum pump capable of a minimum absolute pressure of about $3 \mathrm{kPa}$. In spite of the poor vacuum applied, these two tensiometers later exhibited a satisfactory performance in terms of measurement precision (tensiometers pr3 and pr4 in Tarantino \& Mongiovì, 2000, 2001) that is comparable to that of the best-saturated Trento tensiometers (precision of about $\pm 3 \mathrm{kPa}$ relative to the average measured value). The experience of the discusser is that saturation of the ceramic is achieved mainly through cycles of cavitation and subsequent pressurisation (Tarantino \& Mongiovì, 2001; Tarantino, 2003). An inadequate initial saturation simply increases the number of cycles necessary to obtain a satisfactory performance.

The second issue is the reliable measurement of pore water tension. Tarantino \& Mongiovì (2001) and Tarantino (2003) showed evidence that inadequate saturation might result in readings that appear reliable and stable although actually incorrect. The authors have demonstrated this in a very rigorous fashion, and have addressed perhaps the main problem in tensiometer measurement, which is how to discriminate a good measurement from a bad one.

The authors state that 'tensiometer response times under low absolute pressures are an excellent indicator of tensiometer saturation', implicitly referring to the tests shown in Figs 12 and 14. However, in these tests, water in the tensiometer was subject only to positive absolute pressures and for a limited period of time ( $\sim 35 \mathrm{~min})$. The tensiometer response may not be as good as supposed when water is subject to tension (gauge pressures less than $-100 \mathrm{kPa}$ ) and/ or when measurement lasts for longer periods (from hours to days). Under these conditions, air cavities may form within the tensiometer without triggering cavitation, but causing a faulty response of the instrument. Cavity formation in the tensiometer is illustrated by the test shown in Fig. 16, where the pressure recorded by an Imperial College tensiometer is plotted against time. The ceramic was wiped after about $11 \mathrm{~min}$ to let water pressure drop to about $-1900 \mathrm{kPa}$, and then the tensiometer was immediately replaced in free water. The time required for the tensiometer to return to atmospheric pressure was about $0.5 \mathrm{~min}$ (Fig. 16(b)).

A kaolin paste was then applied to the porous ceramic, and the paste was allowed to dry out slowly. After reaching a pressure of about $-2600 \mathrm{kPa}(595 \mathrm{~min})$ the tensiometer was again replaced in free water (Fig. 16(c)). The equalisation time was much larger in this case, being about $7 \mathrm{~min}$. The reduced permeability is probably due to the expansion of the air cavities in the porous ceramic. Here, it was necessary to reach a very high tension to expand the cavities, but lower tensions might be sufficient to enlarge 

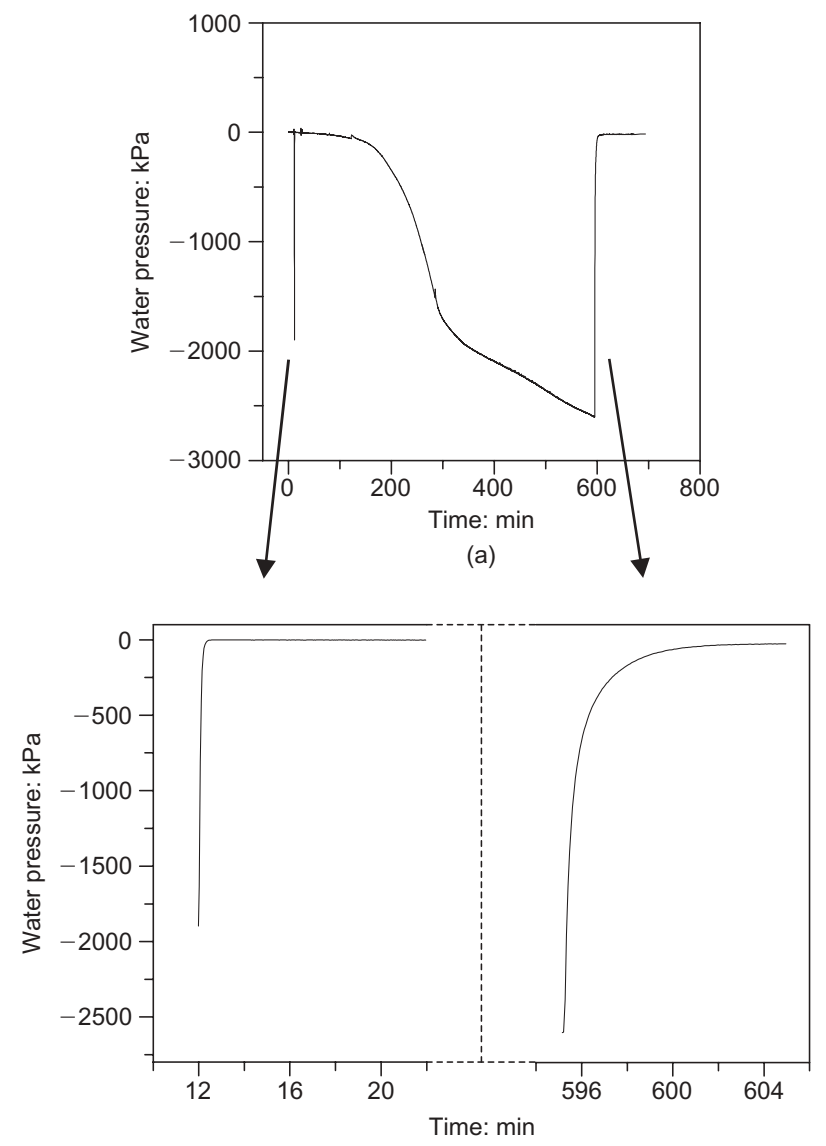

(b)

(c)

Fig. 16. (a) Response of the tensiometer associated with slow drying of the paste applied to the porous ceramic; (b) equalisation in free water before measurement; (c) equalisation in free water after measurement

cavities if the initial degree of saturation of the ceramic is low. These cavities may then cause false tensiometer readings.

Air cavities may expand not only under tension but also under high positive pressures. Tarantino \& Mongiovì (2001) reported the case of a tensiometer stored for 1 year in a saturation chamber under a pressure of $4 \mathrm{MPa}$. After removal from the chamber, the tensiometer could sustain a tension of $650 \mathrm{kPa}$ for some seconds only. However, the same tensiometer could sustain very high tensions (greater than $2000 \mathrm{kPa}$ ) after just one cycle of cavitation and subsequent pressurisation at $4 \mathrm{MPa}$. Despite the high positive pressures, air cavities had expanded in the tensiometer when stored for 1 year because of air diffusion.

These two examples suggest that the degree of saturation of the ceramic must be regarded as a 'dynamic' variable, in the sense that it might change with time. As a result, the types of test shown in Figs 12 and 14 may or may not be indicators of adequate saturation for the case where longer measurements are carried out and/or a tension is applied to water.

To verify the reliability of suction measurement, Tarantino \& Mongiovì (2001) and Tarantino (2003) have suggested indicators of adequate saturation to be checked before and after measurement. These indicators were defined on the basis of long-term measurement using more tensiometers placed on the same sample. Nonetheless, we consider that the straightforward way to verify measurement reliability is to measure suction using at least two tensiometers simultaneously and checking that they give the same readings.

\section{Authors' reply}

The authors would like to thank Dr Tarantino for his interest in the paper. The discusser has provided useful additional information regarding the independent development of a similar technique for the initial saturation of tensiometers. Although motivated by different intentions-a very dry filter element in the authors' case and the minimisation of the absolute pressure at saturation by the discusser-these two techniques share the key features of a dried filter element and the evacuation of the tensiometer in the absence of water. Despite providing a higher degree of initial saturation, the discusser has debated the merits of these techniques as 'inadequate initial saturation simply appears to increase the number of cycles necessary to obtain a satisfactory performance of the instrument'.

This difference in opinion perhaps arises from the differences in the magnitude of the positive pressures used in the second stage of saturation. One objective of the paper was to investigate the process of saturation to permit the measurement of soil suction with more sensitive, low-pressurerange devices that cannot be subjected to large saturation pressures. For example, one version of the tensiometer designed specifically for the accurate measurement of low suctions has a full-scale range of $100 \mathrm{kPa}$. Even if a portion of the safe over-pressure range is used to apply a saturation pressure of $200 \mathrm{kPa}$, a consideration of the physics of saturation (equation (2)) indicates that the initial degree of saturation, $S_{\mathrm{i}}$, must be greater than 0.96 to saturate the ceramic filter sufficiently. In contrast, saturation using positive pressures of the order of $4 \mathrm{MPa}$ (e.g. Tarantino \& Mongiovi, 2001) is likely to be capable of saturating all but the most poorly saturated of devices, given time. Thus it follows from equation (2) that the significance of the high degree of initial saturation will be diminished if very large positive pressures are applied to saturate the device.

The typical pressure range of the base device upon which the new tensiometer is built is $700 \mathrm{kPa}$. At the higher saturation pressure of $1 \mathrm{MPa}$, the initial degree of saturation will be less important, but it is the experience of the authors that the higher degree of saturation ensures the saturation of a device fitted with a 3 bar air-entry ceramic on the first short pressurisation. When saturating up to a dozen of these devices for inclusion into model tests, the authors are of the opinion that this benefit alone is worth the extra effort of ensuring a high initial saturation.

Second, the discusser raises the important issue of stable bubble growth in the ceramic filter element under high absolute tensions, and questions whether tensiometer response times under low absolute pressures are therefore useful indicators of tensiometer saturation. This scenario has been investigated in a further set of experiments using a tensiometer fitted with a nominal 3 bar air-entry ceramic filter element. This tensiometer was initially saturated in the two-chamber saturation apparatus before being subjected to a saturation pressure of $1 \mathrm{MPa}$.

Following the experimental methodology described in the paper, the response time of the instrument was then assessed by subjecting the device to $10 \mathrm{kPa}$ pressure increments of $100 \mathrm{~s}$ duration between 0 and $-100 \mathrm{kPa}$, and between -100 and $0 \mathrm{kPa}$. The device was then removed from the chamber and allowed to generate a pressure of $-250 \mathrm{kPa}$ under the action of evaporation (Fig. 17(a)). At this point, the device was submerged in free water and allowed to equilibrate to atmospheric pressure before the response time was again assessed.

The lower half of this stepwise calibration exercise is shown in Fig. 17(b). The response of the device was observed to be indistinguishable from that observed before the generation of the absolute tension, and-as shown in the 


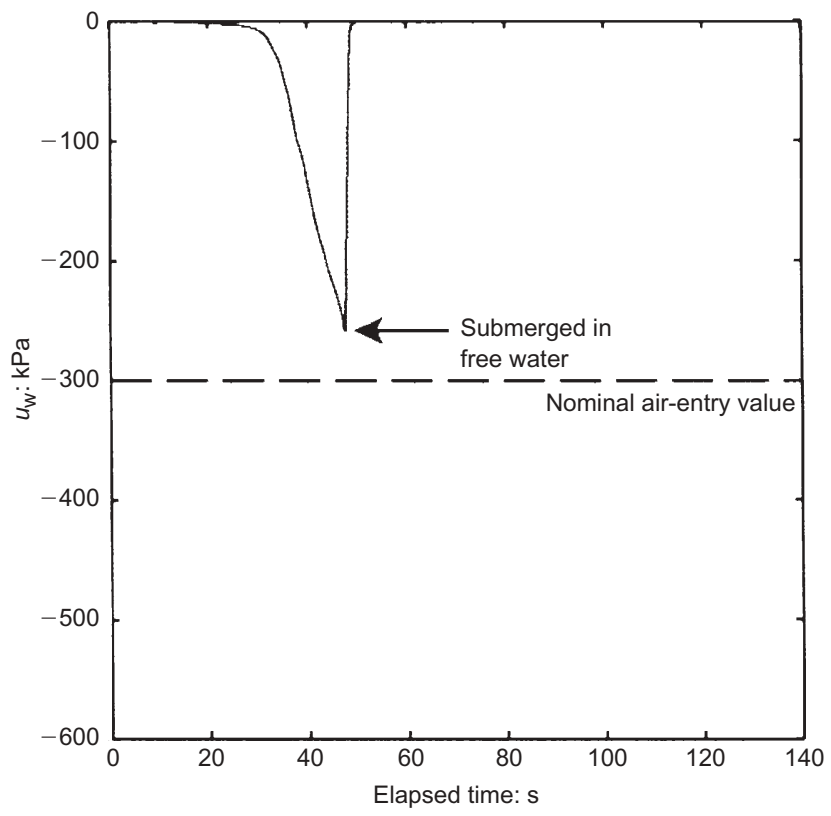

(a)

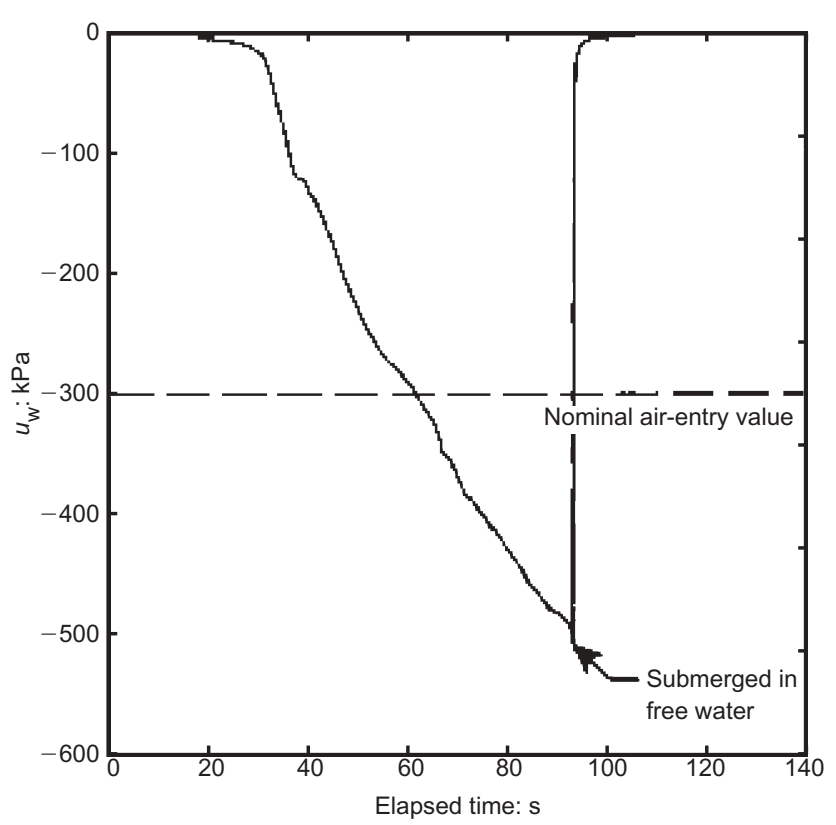

(c)

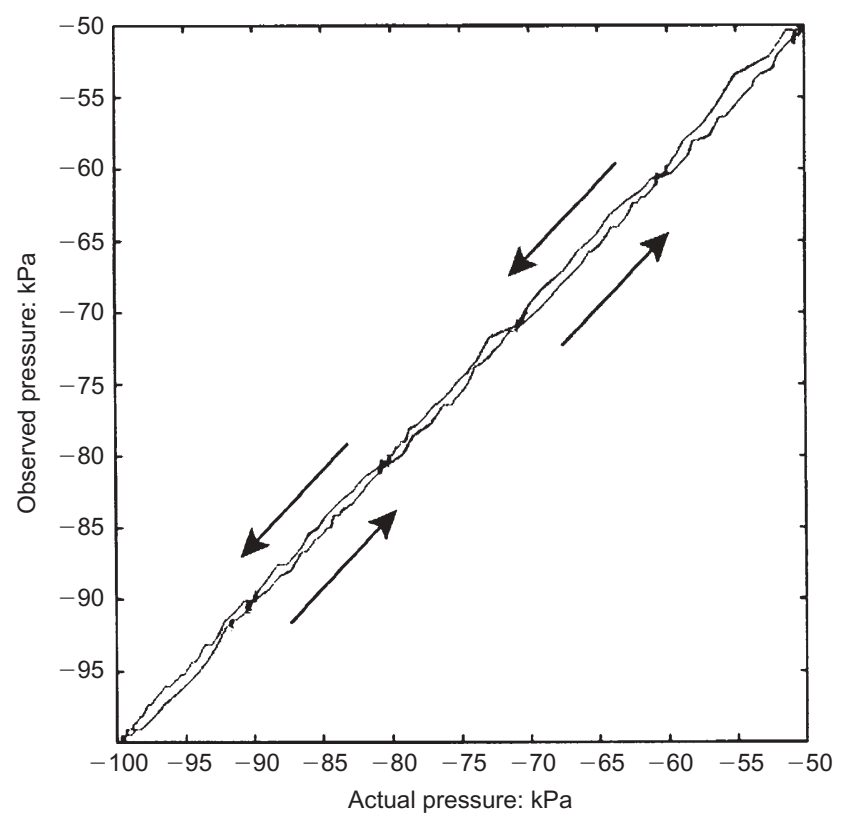

(b)

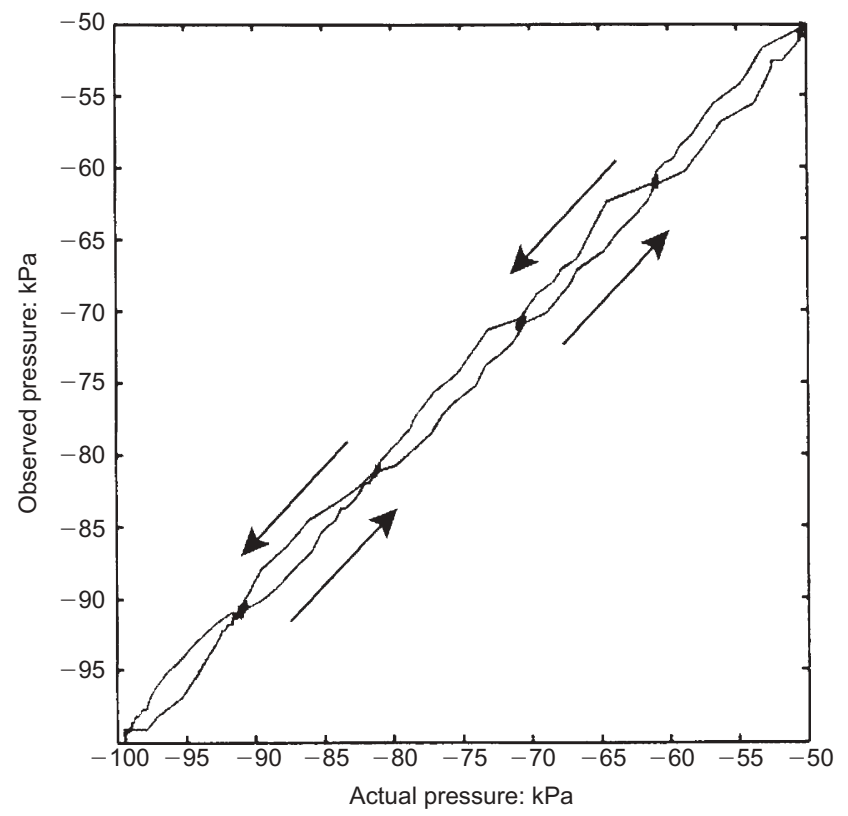

(d)

Fig. 17. Reduction in tensiometer response times with stable air entry

figure-indicates little lag-time observed between the applied and measured pressures. The device was then subjected to a further pressure of $-500 \mathrm{kPa}$ by evaporation (Fig. 17(c)), before being plunged into free water. Upon pore pressure equalisation, the device was again assessed for its response time under low absolute pressures. These results, presented in Fig. 17(d), indicate that even the short-term generation of this higher tension has reduced the permeability of the filter element, despite not having initiated nucleation.

Rather than the absolute magnitude of the applied tension, the authors would suggest that it is the fact that the air entry value has been exceeded that gives rise to this response, and that reported by the discusser (Fig. 16). Although the air-entry value of a ceramic is often quoted as a single value, the pore size distribution will ensure that air entry is not an instantaneous process. Rather, as the tension is increased past the nominal air-entry value, the menisci at the exposed surface of the tensiometer will start retreating into the ceramic, beginning with the largest of exposed pores. The thicker the filter element, the longer this process can continue before nucleation occurs, the more unsaturated the surface of the ceramic can become, and the larger the tension that can be recorded in the reservoir of the device. The difference in response time observed by the discusser is a result of a longer period of desaturation. This observation, in combination with the experiment described herein, indicates that largest measurable tension in the reservoir should not be the defined as the largest measurable suction, as applied suctions greater than the air-entry value of the ceramic will initiate the process of desaturation. As the process of desaturation will always be accompanied by a reduction in permeability, any technique evaluating this property either quantitatively or qualitatively (as in the technique proposed by the authors) will provide a good indication of tensiometer saturation. 


\section{REFERENCES}

Ridley, A. M. \& Burland, J. B. (1999). Discussion: Use of tensile strength of water for the direct measurement of high soil suction. Canadian Geotechnical Journal, 36, 178-180.

Tarantino, A. (2003). Panel report: Direct measurement of soil water tension. Proc. 3rd Int. Conf. on Unsaturated Soils, Recife 3 (in press).

Tarantino, A. \& Mongiovì, L. (2000). Experimental investigations on the stress variables governing unsaturated soil behaviour at medium to high degrees of saturation. In Experimental evidence and theoretical approaches in unsaturated soils (eds A. Tarantino and C. Mancuso), pp. 3-19. Rotterdam: Balkema.

Tarantino, A. \& Mongiovì, L. (2001). Experimental procedures and cavitation mechanisms in tensiometer measurements. Geotechnical and Geological Engineering 19, Nos 3-4, 189-210.

Tarantino, A. \& Mongiovì, L. (2002). Design and construction of a tensiometer for direct measurement of matric suction. Proc. 3rd Int. Conf. on Unsaturated Soils, Recife 1, 319-324. 